\title{
High rate loading of hybrid joints in a Split Hopkinson Tension Bar
}

\author{
Noah Ledford ${ }^{1 *}$, Hanna Paul $^{1}$, Matti Isakov ${ }^{1}$ and Stefan Hiermaier ${ }^{1}$ \\ ${ }^{1}$ Fraunhofer Institute for High-Speed Dynamics, Ernst-Mach-Institut, EMI, Freiburg, Germany
}

\begin{abstract}
Bonded joints are nowadays seen as one of the preferred joining methods in aerospace applications. However, the difficulty in certifying bond strength and the relatively low energy absorption capability of the joint are barriers to widespread adoption. The use of a hybrid joint, that is, the combination of a mechanical and a bonded joint, allows for a fail-safe design and offers improved performance of the joint. The quasi-static properties of hybrid joints have been investigated by a number of researchers. In contrast, the high rate loading regime has been only sparsely investigated. In this work, hybrid joints are tested in quasi-static and high rate loading in order to analyze their loading rate dependence. The hybrid joint studied is a composite-aluminum double lap shear joint with Sikaforce 7752 adhesive and Hi-Lite-315 countersunk titanium bolts. In order to quantitatively analyze the high rate behavior of the hybrid joints and their respective sub-components, additional tests are carried out on simply bonded and simply bolted specimens. The high rate characterization was performed with a Split Hopkinson Tension Bar. The main challenges for these tests are the relatively large specimen size and complex specimen geometry needed to properly characterize the joint behavior, which both are in contradiction with the assumptions of the classical Split Hopkinson Bar -analysis. In this paper we describe an approach to solve these challenges based on an elastic wave analysis of the system.
\end{abstract}

\section{Introduction}

The ever increasing demand for more efficient structures is bringing multi-material design into the technological forefront. The selection of the right material at the right location allows for the simultaneous fulfillment of multiple design objectives. The improved performance comes, however, with the challenge of properly joining different types of materials together to form a structure. There is an increasing awareness that crash and impact loading should be highlighted in the design process and that the joints in a structure play a large role in determining the overall structural response [1].

Hybrid joints, which combine mechanical and bonded joints, offer improvements to strength and energy absorption. This is achieved by load sharing between the two different joining methods. The hybrid joints can also offer many additional benefits such as electrical insulation, water tightness, corrosion protection and improved damping. These types of joints have been investigated by many researchers in the quasistatic and fatigue regime. In a recent review Bodjona et al. [2] highlight the potential of hybrid joints. However, they also underline the need to further study the high rate response of these joints.

Testing of joints at high rates of loading is challenging because of the conflicting requirements between the test methods and the joint specimens to be tested: In dynamic tests a small and simple specimen geometry is preferred so that an (quasi-) equilibrium of forces within the specimen is achieved during the test. This is required for a representative measurement of the force acting on the specimen. In contrast, the joints used in the industry tend to be relatively large in size and have relatively complex geometry, such as several interfaces between different materials. It should be noted that since joints are essentially mechanical structures, they cannot in general be simplified or reduced in size without affecting their loading response.

Mechanically fastened joints have been tested at elevated rates of loading by many different authors [3-9]. Based on these studies it can be concluded that the geometry and the material of the components being joined play a major role in determining the failure mode of the joint. This, in turn, often determines whether rate dependence is seen in the joint response. For example, some studies $[3,4,6,8,9]$ report a significant increase in dynamic strength or energy absorption, whereas some studies indicate no loading rate effect $[5,7]$. This highlights the importance of testing the joint in a configuration as near as possible to the intended application.

Adhesive bonded joints generally show significant rate dependence, which has to be accounted for in structures where crash and impact may take place [1013]. Similarly to the mechanical joints, also adhesive joints have to be considered as structures in testing. That is, the specimen geometry, the materials being joined,

Corresponding author: Noah.Ledford@emi.fraunhofer.de 
and the surface preparation play a role in determining the mechanical response.

In contrast to mechanical and adhesive joints, hybrid joints have been tested in the dynamic regime by only few researchers. Graham et al. [14] carried out experiments on pinned/bonded joints and showed significant rate dependence in both the maximum force carried by and the energy absorbed by the joint. Other works on hybrid bolted/bonded joints [15-16] also report a positive rate sensitivity of the maximum force carried by the joint.

As noted above, due to their structural complexity joint specimens introduce challenges in high rate tests. Up to date, most high rate tests on mechanical joints have been performed using high speed servo-hydraulic machines [4-6, 8, 14]. This test method has the advantage of being flexible in terms of what can be tested, such as the capability to impart high forces on a large specimen over an extended time period (leading to large displacement). The main drawback of servohydraulic machines is that at high enough loading rates the dynamic structural response (natural vibrations) of the test machine itself starts to influence the force measurement. In this case one is faced with the cumbersome task of separating the structural response of the specimen from the structural response of the test machine. This task has to be often carried out based on a single load signal measured far away from the specimen.

In contrast to high speed servo-hydraulic machines, in the Split Hopkinson Bar (SHB) technique the overall test setup geometry is very simple and the characteristic vibrations of the test frame (the elastic stress waves in the bars) are used as a basis of the force and displacement measurement. The boundary conditions imparted on the specimen are well known throughout the test and the measurement signals can be related to the specimen response with relative ease and minimal need for data filtering. The main drawback of the Split Hopkinson Bar technique are the strict requirements in the maximum allowable force and boundary velocity imparted on the specimen as well as the limited loading duration (typically max. $100 \mathrm{kN}$ at $\max .20 \mathrm{~m} / \mathrm{s}$ can be imparted on the specimen for max. $1 \mathrm{~ms}$ ).

There are some reports in the literature on the testing of mechanical and hybrid joints with the SHB technique $[3,7,9,15-17]$. Based on these works it seems that high rate testing of joints with the SHB technique is feasible, but generally accepted methods are still lacking. In the current contribution we report high rate loading experiments on mixed material hybrid joints by using the Split Hopkinson Tension Bar (SHTB)-technique. We focus on the test method, that is, on the design of the specimens, test parameters and analysis of the raw data. In particular, we highlight the aspects in which the current experiments differ from the classical SHB tests on homogeneous solid materials.

\section{Test specimens}

\subsection{Joint geometry and materials}

The hybrid joint specimen tested in this work is illustrated by Figure 1. The joint is of a double lap sheartype with an aluminum 2024-T3 center plate and two quasi-isotropic carbon-fiber-reinforced composite (CFRP) plates on the outside. The CFRP-plates have a $[45,90,-45,0] \mathrm{s}$ layup and they were prepared from a TenCate Prepreg Laminate (HTS-40 12k Carbon Fiber and TenCate TC250 resin) by Connova $\mathrm{GmbH}$ (Germany). The adhesive used in the joint is SikaForce 7752 L60 (a flame retardant two component polyurethane structural adhesive). The mechanical part of the joint consists of a countersunk Titanium Hi-Lite fastener with a $4 \mathrm{~mm}$ diameter HST-315-5 bolt with a HST-71 collar.

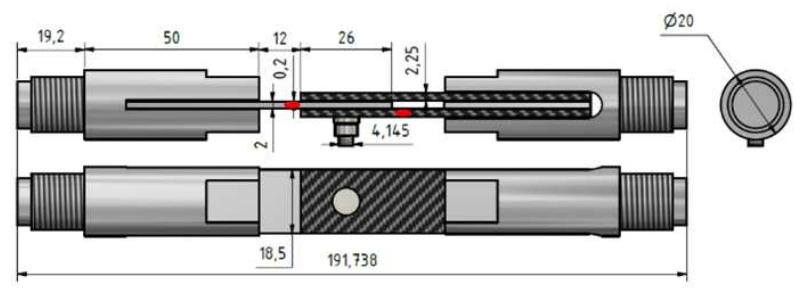

Fig. 1. The geometry of the hybrid specimen and the mountings between the specimen and the bars of the SHTB setup. The location of the local displacement measurement by means of Digital Image Correlation are shown by the red ellipses.

Based on literature, the SikaForce 7752 adhesive has a shear modulus of $0.190 \mathrm{GPa}$, yield and ultimate strength of $5.1 \mathrm{MPa}$ and 10.2 MPa, respectively, and a failure strain of 0.55 [18]. Based on manufacturer data, the Hi-Lite mechanical fastener is expected to fail in double lap shear at $17.8 \mathrm{kN}$. The aluminum (2024-T3) is a standard aerospace material with limited strain rate dependence. The reported elastic modulus, yield point, and failure stress are $73.1 \mathrm{GPa}, 347 \mathrm{MPa}$ and $476 \mathrm{MPa}$, respectively [19]. Based on in-house tensile tests, the composite material has an elastic modulus of $55.5 \mathrm{GPa}$ and failure stress and strain of $799 \mathrm{MPa}$ and 0.014 , respectively.

A double lap joint was chosen for two reasons. The first is to avoid the bending moment effect taking place in a single lap joint. Secondly, the chosen specimen geometry allows for focusing on the failure modes of the adhesive and the aluminum component. That is, the double-plate side of the specimen (right-hand side in Figure 1) is made of the CFRP to make sure that failure takes place in the aluminum component. As noted above, the selected aluminum alloy shows very little strain rate dependence, which allows for the rate dependence of the joint itself to be measured (the CFRP plates are assumed to deform only elastically). Based on the dimensions presented in Figure 1 the joint has edge/diameter and width/diameter ratios of 2.8 and 4.6, respectively. These values are typical in aircraft applications and should lead to failure sequence of 1 . adhesive failure and 2 . bearing/net tension failure in the aluminum plate.

In addition to the hybrid joint specimens, specimens with purely mechanical and purely adhesive joint were tested. The basic geometry of these specimens 
is the same as depicted in Figure 1. Furthermore, the mechanical joint specimen is expected to fail by a bearing/net tension failure of the aluminum plate similarly to the hybrid specimen.

\subsection{Specimen preparation}

The joints in the specimens were prepared by using the jig shown in Figure 2. All bond surfaces were prepared by cleaning with isopropanol, mechanical abrasion with 400 grit sandpaper, and a second round of cleaning with isopropanol. This was followed by a low pressure plasma cleaning treatment for 5 minutes. Teflon spacers were used to define the adhesive thickness of $0.2 \mathrm{~mm}$. This selection allows the use of literature data for the adhesive properties. After applying the adhesive and assembling the aluminum and composite plates together the joint was cured at room temperature for two days. After that a second jig was used to bond the SHTBmounting parts (shown in Figure 1) into the specimen. This bonding was done with a structural adhesive (ScotchWeld DP-490) and cured at $65^{\circ} \mathrm{C}$ for two hours. The mounting components are made of 7075 aluminum and thread into the bar ends of the SHTB setup.

It should be noted that the bolt component of the mechanical fastener was installed during the first bonding step but the collar of the bolt was not installed until after the final curing. This approach was chosen in order to make sure that the pre-defined adhesive thickness is obtained. During the installation of the collar used in the Hi-Lite connection, a part of the bolt shears off at a given prescribed torque level (nominally $2.3 \mathrm{Nm}$ ). This results in a reproducible preload in the bolt.

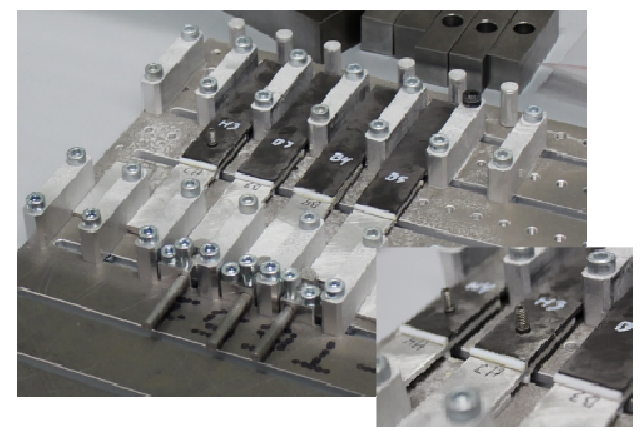

Fig. 2. Jig for the specimen preparation.

\section{Testing methods}

The high rate testing was performed using a Split Hopkinson Tension Bar. During the test a $2.4 \mathrm{~m}$ long aluminum striker is accelerated and impacted on the flange at the end of the input bar, which generates an incident tensile wave with a duration of $1 \mathrm{~ms}$. The input bar (titanium, Ø20 mm) consists of two components coupled with a bolted connection. As will be discussed later, a small part of the incident wave is reflected back at the connection, which causes an artifact on the measurement of the stress waves but does not affect the specimen loading itself. In total the length of the input bar is 8.8 meters. The output bar of the setup is $3.1 \mathrm{~m}$ long (titanium, Ø20 mm). The specimen is attached with the mountings described above into the M16x1.5 threads in the bar ends.

The elastic stress waves in the bars are measured using strain gauges bonded on the input and output bars at a distance of $2.796 \mathrm{~m}$ and $0.301 \mathrm{~m}$ from the specimen, respectively. The deformation in the bars is assumed to be linear-elastic. The strain gauge signal readings are verified quasi-statically in terms of calculated force by using a calibrated load cell mounted between the bars. In the analysis of the SHTB tests the measured strain gauge signals are time-shifted by assuming a constant propagation velocity of the elastic stress waves. Dispersion of the high rate components of the stress waves is neglected in this paper. During the tests the strain gauge signals are recorded at $10 \mathrm{MHz}$ using a digital oscilloscope. The results presented in this paper are based on unfiltered signals.

During the high rate tests the specimen was monitored with a high speed camera (Phantom 1610 at $150 \mathrm{kHz}$ and $1024 \times 96$ pixels) allowing for local strain and displacement measurements based on the 2D Digital Image Correlation (DIC) technique. For the deformation tracking a black-and-white stochastic speckle pattern was laid on the specimen surface using water soluble spray paint. The DIC analysis was carried out with the GOM Correlate 2016 software with a subset size of 9 pixels corresponding to $\sim 2$ millimeters.

At the beginning of a high rate test both the digital oscilloscope and the high speed camera record in a continuous buffer-mode. The oscilloscope is triggered by the incident wave arriving at the input bar strain gauge station. The oscilloscope sends a trigger signal to the camera, which then sends a respective feedback signal to the oscilloscope thus ensuring a precise synchronization of the two devices. Before the actual experiments the operation of the setup and the timeshifting of the stress waves was verified by carrying out tests without the specimen and comparing the bar-end displacement obtained from the wave analysis against the one measured with the DIC technique.

In this paper the joint specimens were tested at a relative bar end velocity of $10 \mathrm{~m} / \mathrm{s}$.

In addition to the high rate tests, quasi-static tests were carried out with a servo hydraulic materials testing machine Instron 8800 at a velocity of $10^{-5} \mathrm{~m} / \mathrm{s}$. The force acting on the specimen was measured with a $50 \mathrm{kN}$ load cell and the displacement with a LVDT-transducer attached on the machine actuator. In addition, low rate digital cameras were used to track the specimen deformation. In the low strain rate tests the specimen was mounted directly into the grips of the testing machine without the bonded mounting components.

\section{Theoretical analysis of the high rate tests}

As noted above, the specimens studied in this paper differ from the typical specimens used in high strain rate testing. The main guideline of the approach presented in this paper is that no a priori assumptions on force or 
deformation equilibrium in the specimen are made. Instead, the specimen is considered to be a structure, which is imposed to a fixed-length uniaxial loading pulse and its response is then measured. As is shown later by the results, the SHB technique is well suited for this kind of measurement.

In the following analysis it is assumed that the bars of the SHTB setup and the mountings between the specimen and the bars remain linear elastic throughout the loading event. The specimen is also assumed to behave linear-elastically upon the first passing of the initial loading wave. For simplicity, the system is analyzed based on the assumption of uniaxial loading and wave propagation.

The specimen with the mounting components attached has multiple interfaces, where the impedance $Z$ changes abruptly due to changes in geometry and/or material properties ( $Z=$ cross-sectional area $A$ x Young's modulus $E$ / elastic wave speed $C$ ). These interfaces will lead to reflections of the incident wave, as depicted by the Lagrange diagram in Figure 3 (dashed green curves). As can be seen, wave reflections are expected to take place at the bar/mounting-interfaces, within the mountings, at the mounting/specimen-interfaces and within the specimen. From this list only the last one, the reflections within the specimen, are of interest from the results point of view. Moreover, it is noted that the largest change in impedance within the mounting takes place at the bar/mounting interface (a decrease by $\sim 50 \%$ ). Therefore the complex wave motion within the mountings is modelled with a simplified model depicted by the solid orange curves in Figure 3 .

The impedance of the mountings in the model is obtained by a linear rule of mixtures approach at the location where the specimen plates are bonded into the mounting. In addition, the mountings are assumed symmetric in the model.

This simplification of the system allows for a straightforward calculation of the transmission and reflection of the incident wave taking place within the mountings. In other words, the forces acting on the specimen are determined at the mounting/specimen interface ( $\mathrm{B}$ and $\mathrm{C}$ in Figure 3 ) by taking into account the effect of the mountings on the measured stress waves.

The numerical approach used here is based on the solution of the uniaxial elastic stress wave propagation in the presence of multiple interfaces (see for example [20]). In short, the equations for particle velocity (Equations 1 and 2) and cross-sectional force (Equations 3 and 4) are solved for each interface (" $A$ " to " $D$ " in Figure 3) to obtain the amplitudes of the waves transmitted through and reflected at the interface. These waves are then time-shifted to the next interface(s), where the calculations are repeated. It should be noted that the wave motion can be solved equally forwards and backwards in time.

On the input bar side the measured incident and reflected waves are used as input data for the calculation of the specimen force, whereas on the output bar side the measured transmitted wave is used. All the calculations were carried out by using the Matlab software.

\section{Input bar Mounting Specimen Mounting Output bar}

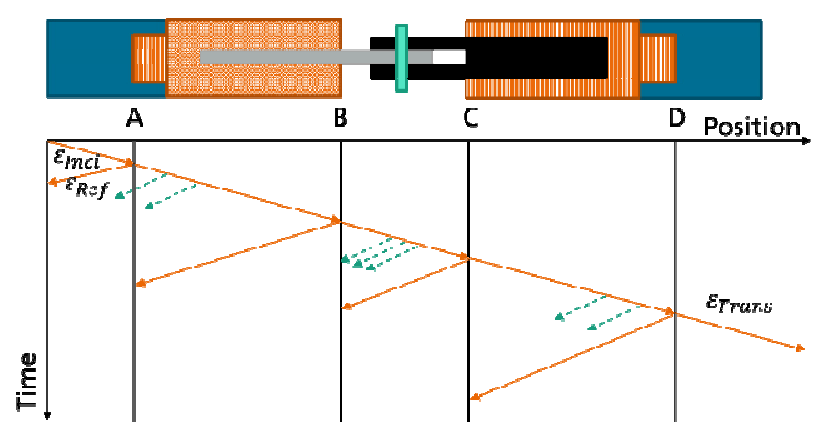

Fig. 3. Lagrange diagram of the specimen with mountings (green dashed curves) and that assumed in the model (orange curves).

$$
\begin{aligned}
& V_{i}=-C_{\mathrm{i}}\left(\varepsilon_{i}\right) \\
& V_{\text {int }}=-C_{A}\left(\varepsilon_{\text {Ain }}-\varepsilon_{\text {Aout }}\right)=-C_{B}\left(\varepsilon_{\text {Bout }}\right) \\
& F_{i}=E_{i} A_{i} \varepsilon_{i} \\
& \sum F_{\text {int }}=E_{A} A_{A}\left(\varepsilon_{\text {Ain }}+\varepsilon_{\text {Aout }}\right)-E_{B} A_{B} \varepsilon_{\text {Bout }}=0
\end{aligned}
$$

where $V_{i}$ and $F_{i}$ denote the particle velocity and the force caused by a propagating uniaxial elastic wave, respectively. The strain caused by an elastic wave is denoted by $\varepsilon$. Equations (2) and (4) describe the arrival of a single incident wave on an interface with a change of properties from " $A$ " to be "B". The arrival of waves simultaneously from both sides can be solved by adding the corresponding strain term on the "B" side.

The analysis procedure was tested by reloading a failed joint-specimen (Figures 5 and 6). In this case it can be assumed that the calculated specimen load should be close to zero due to the free end condition on the specimen. A mounting with a failed specimen was selected instead of a mounting only in order to account for the effect of the specimen material within the mounting on the stress wave propagation.

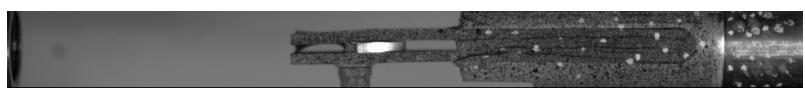

Fig. 4. Reloading of a failed joint specimen (input bar is seen on the right, the output bar is not connected to the specimen).

The waveforms presented in Figure 5 were timeshifted to the bar/mounting interfaces based on the initial experiments with bars only (i.e., no mounting or specimen) and no subsequent shifts were applied. As can be seen in Figure 5, the initial slopes of the incident and reflected waves and the without correction calculated force (raw sum of the waves) do not agree well with the free-end condition on the specimen: the slope of the reflected wave is notably smaller than that of the incident wave. This leads to notable peaks in the calculated force at the beginning and end of the loading. In contrast, the proposed correction methodology notably reduces these peaks in force. This happens because the correction algorithm takes into account the reflections within the mountings, i.e., the inertia of the mountings. It should be noted that one cannot improve the raw solution for the force (dotted green curve in Figure 5) by adjusting the time-shift because of the 
differing slopes of the incident and reflected wave. As can be seen in the Figure 5, the correction method does not fully remove the peaks in the calculated force. This is expected since the mounting geometry is simplified in the model and because the model neglects the part of the specimen remaining outside the mounting (seen in Figure 4).

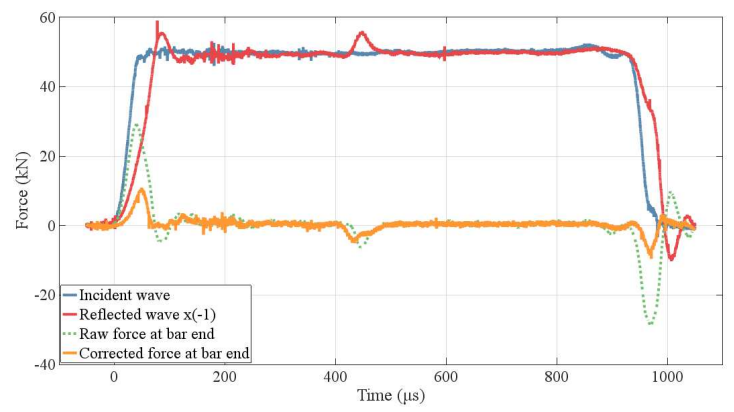

Fig. 5. Measured waves (time-shifted) and calculated bar end force (with and without correction for the mounting effects) in the reloading test of a half of a joint specimen.

Finally, an unexpected peak in the reflected wave (and in the calculated forces) is observed in Figure 5 around $450 \mu$ s. As was noted above, this is an artifact in the measurement of the reflected wave. The coupling element between the two parts of the input bar causes a small part of the incident wave to be reflected back as a wave of tension. This short-duration wave travels to the free end of the input bar and reflects back as a wave of compression, then travels through the connection (with again partial reflection) and arrives at the strain gauge location at the same time as the reflected wave coming from the specimen. Therefore, the peaks seen in the reflected waves presented in this paper at $450 \mu \mathrm{s}$ after the start of loading should not be related to specimen behavior.

\section{Results and discussion}

Quasi-static and high-rate tests were conducted to measure the response of the joint described above. The bonded and mechanical joints that make up the hybrid joint were also tested individually to see how the different components behave under different loading rates.

Figure 6 presents the unfiltered time-shifted incident, reflected, and transmitted waves measured in the high rate SHTB tests for each joint type. As can be depicted from the reflected and transmitted waves, each joint fails during the first incident wave. Furthermore, the time delay between the incident and transmitted waves caused by the wave propagation through the long specimen/mounting-structure is clearly visible in Figure 6 (the time-shift moves the waves to correspond to the bar/mounting interfaces).

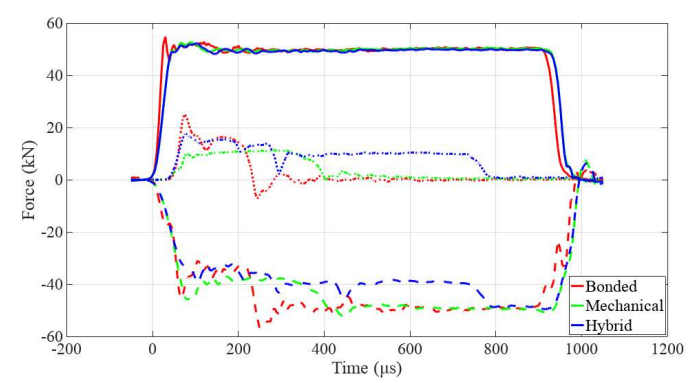

Fig. 6. Time-shifted incident (solid lines), reflected (dashed lines), and transmitted (dotted lines) waves measured in the SHTB tests of the three joint types.

Figure 7 illustrates the effect of the above discussed correction algorithm on the calculated specimen forces in the case of the hybrid joint specimen. The initial oscillations on the input and output force are notably reduced by the application of the correction algorithm. Furthermore, the correction results in a realistic "timeshift" of the forces, i.e., input force is shifted $\sim 10 \mu \mathrm{s}$ forward in time (corresponding to wave travel through the mounting) whereas the output force is shifted by a similar amount backwards in time. It should be noted that these "time-shifts" are not explicitly included in the algorithm but result implicitly from the calculated wave motion and reflections within the mountings. As a result, the time-offset between the forces (depicted based on the initial portions of the curves in Figure 7) corresponds after the correction to the elastic wave travel time through the specimen, as expected. Furthermore, since the calculations do not involve any filtering, all the information carried by the elastic waves is preserved.

Figure 7 also allows for the estimation of force equilibrium in the specimen, which takes place at around $120 \mu \mathrm{s}$ (the first adhesive layers fails around $175 \mu \mathrm{s}$ ). Similar conclusions can also be made in the case of other specimen types. As noted above, the artifact caused by the coupling element in the input force is visible around $450 \mu$ s in Figure 7.

Figure 8 summarizes the results of the high rate tests by plotting the calculated specimen force at the output bar-side as a function of the relative displacement obtained from the DIC analysis (location of the tracking points is indicated in Figure 1). In addition, the results of the quasi-static tests are presented in the Figure 8 in terms of force measured by the load cell versus relative local displacement obtained with the DIC method.

The results of both the quasi-static and the high rate tests are in accordance with the initial predictions. The first drops in load in the hybrid joint tests (around $0.4 \mathrm{~mm}$ in the quasi-static curve and around $1 \mathrm{~mm}$ in the high rate curve in Figure 8) correspond to the initiation of failure of the double lap adhesive joint. The load levels in these tests are in agreement with the corresponding tests of the simply bonded joints (red curves). The partial bond failure in the hybrid joint is followed by the complete failure of the adhesive (around $0.9 \mathrm{~mm}$ and $1.8 \mathrm{~mm}$ in quasi-static and high rate tests, respectively). Then the load is transferred to the mechanical component of the joint. Also in this case the 
load carried by the hybrid joint matches well the load carried by the purely mechanical joint (green curves).

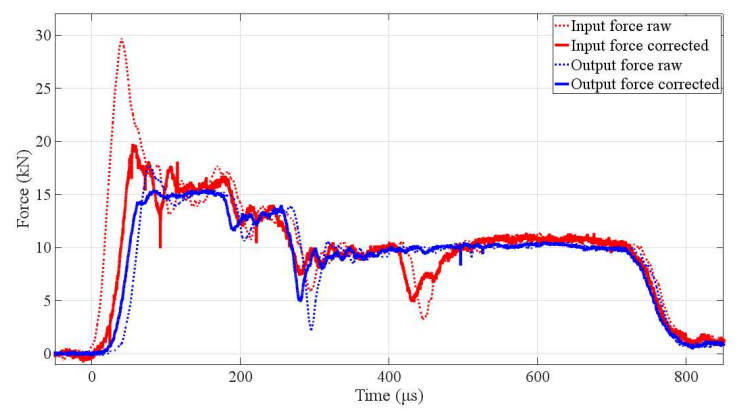

Fig. 7. Calculated forces acting on the specimen in a SHTB test of a hybrid joint (with and without correction for the mounting effects).

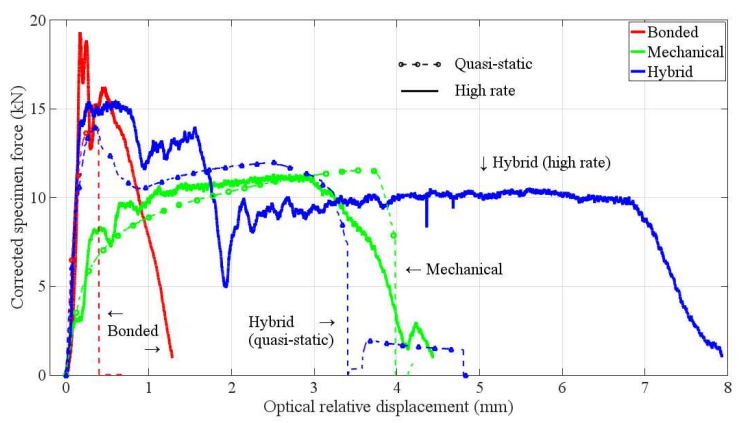

Fig. 8. Quasi-static and high rate force versus displacement response of the joints.

The high rate load-displacement curve of the mechanical joint in Figure 8 shows initial oscillations caused by the play in the mechanical joint. After this the aluminum plate starts to deform plastically before failing in a bearing/shearing mode. After the failure of the aluminum plate some force is carried through the joint by frictional forces caused by the pre-load of the bolt compressing the parts together. Similar conclusions can be made in the case of the hybrid joint.

Comparison between the quasi-static and high rate tests (Figure 8 ) indicates relatively small loading rate effect in the tested joints. The maximum load carried by the purely adhesive joint increases somewhat with increasing loading rate whereas the load carried by the mechanical joint appears loading rate independent. The load carried by the hybrid joint after the adhesive failure seems to decrease with increasing loading rate. In terms of displacement to complete failure, the mechanical joint shows little loading rate dependency. In contrast, the both the bonded and the hybrid joint show considerably larger displacement to failure in the high rate test. The boundary conditions for the two tests are notably different: the transverse stiffness of the quasi-static tensile testing machine is considerably higher than that of the SHTB-setup. This may contribute to the specimen behavior after the initial bond failure depending on to which degree the specimen is free to rotate/move laterally in the case of asymmetric deformation/local failure. This feature is subjected to further studies and therefore the presented comparison between the loading rates should be considered preliminary.

\section{Conclusions}

A framework for performing high rate tests on hybrid joints using a Split Hopkinson Tension Bar was presented in this paper. An analysis method was applied to account for the elastic wave distortion caused by the specimen mountings. It was shown that the high rate response of a multi-material hybrid joint can be measured in detail using the proposed methodology. This was evidenced by the ability of the technique to detect the step-wise failure of the hybrid-joint.

The Authors would like to thank the personnel from Airbus Deutschland and WIWeB for technical consulting regarding the joint design and Sika Deutschland for providing the adhesive.

\section{References}

1. S. Heimbs, M. Hoffmann, M. Waimer et al. Int. J. of Crashworthiness 18:406-22 (2013)

2. K. Bodjona, L. Lessard. J. of Reinforced Plastics and Composites 35:764-81 (2016)

3. M. Daimaruya, H. Fujiki, H. Ambarita et al. J. Phys.: Conf. Ser: 451 (2013)

4. B. Egan, C. McCarthy, M. McCarthy et al. Composites Part A 53: 97-108 (2013)

5. S. Heimbs, S. Schmeer, J. Blaurock et al. Composites Part A 47: 91-101 (2013)

6. G. Pearce, A. Johnson, R. Thomson et al. Appl Compos Mater 17: 271-91 (2010)

7. R. Porcaro, M. Langseth, A. Hanssen et al. Int. J. of Impact Engineering 35: 1251-66 (2008)

8. X. Sun, M. Khaleel. Int. J. of Impact Engineering 34: 1668-82 (2007)

9. P. Wang, R. He, H. Chen et al. Composites Part B 79: $322-30$ (2015)

10. G. Challita, R. Othman, P. Casari et al. Int. J. of Adhesion and Adhesives 31: 146-53 (2011)

11. H. Sankar, M. Adamvalli, P. Kulkarni et al. Int. J. of Adhesion and Adhesives 56: 46-52 (2015)

12. M. May, O. Hesebeck, S. Marzi et al. Engineering Fracture Mechanics 133: 112-37 (2015)

13. Neumayer, P. Kuhn, H. Koerber et al. The J. of Adhesion 92: 503-16 (2015)

14. D. Graham, A. Rezai, D. Baker et al. Composites Part A 64: 11-24 (2014)

15. David S. Gonzalez (Master of Science): Michigan State University (2014)

16. Aiswarya Venkadachalam (Master of Science): Michigan State University (2014)

17. A. VanderKlok, A. Dutta, S. Tekalur. Composite Structures 106: 446-52 (2013)

18. T. Faneco, R. Campilho, F. Silva et al. J. Test. Eval 45: 398-407 (2017)

19. J. Seidt, A. Gilat. Int. J. of Solids and Structures 50: 1781-90 (2013)

20. D. Parry, P. Dixon, S. Hodson et al. J. Phys. IV France 04: 107-112 (1994) 